International Journal of Pure and Applied Mathematics

Volume 87 No. 4 2013, 587-602

ISSN: $1311-8080$ (printed version); ISSN: 1314-3395 (on-line version)

url: http://www.ijpam.eu

doi: http://dx.doi.org/10.12732/ijpam.v87i4.8

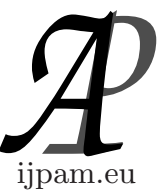

\title{
A STUDY ON UNCONDITIONALLY STABLE EXPLICIT DIFFERENCE SCHEMES FOR THE VARIABLE COEFFICIENTS PARABOLIC DIFFERENTIAL EQUATION
}

\author{
Masaharu Nakashima \\ Kagoshima-Shi \\ Taniyama Chuou 1-4328, 891-0141, JAPAN
}

\begin{abstract}
In [2], we have presented some new algorithms for solving the linear variable coefficients parabolic differential equations. The proposed scheme is stable without any restriction to step-sizes of space and time. The scheme is required the condition of step size ratio $\frac{k}{h^{2}}, \rightarrow 0$ as $k, h \rightarrow 0$ in the convergence, where $k$ and $h$ are step sizes for space and time respectively. In this paper, we will present the explicit unconditional stable scheme which has no restriction on the step size ratio $\frac{k}{h^{2}}$ in the convergence. We will also present analysis for the present scheme.
\end{abstract}

AMS Subject Classification: 65L06, 65L07

Key Words: Runge-Kutta methods, method of lines, difference equation

\section{Introduction}

A number of difference schemes for solving partial difference equations have been proposed. The methods of lines are recognized as useful tools in the numerical solution of PDEs. E.C. Du Fort and S.P. Frankel [1] and some others have proposed difference schemes based on methods of lines. However, in using the explicit lines methods, stability of algorithms is a serious problems. We[2,3]

Received: June 15, 2013

(c) 2013 Academic Publications, Ltd. url: www.acadpubl.eu 
have proposed some explicit difference schemes by using the idea of methods of lines and overcome this problems. The problem considered in [2] is

$$
\begin{gathered}
\frac{\partial u(x, t)}{\partial t}=\frac{\partial}{\partial x}\left(a(x, t) \frac{\partial u(x, t)}{\partial x}\right)+b(x, t) \frac{\partial u(x, t)}{\partial x}+c(x, t) u(x, t), \\
a(x, t)>0 \\
(x, t) \in \Omega=\left\{(x, t) ; 0<x<1,0<t \leq t_{F}\right\}
\end{gathered}
$$

with the initial Dirichlet boundary condition

$$
u(x, t)= \begin{cases}f(t), & (0, t) \in \partial \Omega \cup \Omega, \\ 0, & (1, t) \in \partial \Omega \cup \Omega .\end{cases}
$$

In the proposed scheme [2], it is required the condition of step size ratio

$$
\frac{k}{h^{2}} \rightarrow 0 \quad \text { as } \quad h, k \rightarrow 0
$$

in the convergence, where $h$ and $k$ for space and time respectively. In this paper, we propose the difference approximation to (1.1) where the step size ratio is defined by

$$
\frac{k}{h^{2}}=c \quad(c \text { is any positive constant }) .
$$

The outline of this paper is as follows. In Section 2, by using idea of methods of lines, we present the explicit difference approximation to (1.1). In Section 3 , we study the truncation errors of our scheme. In Section 4, we study the convergence of the scheme with the condition (1.3) and we will show that our scheme converges to the true solution of (1.1). In Section 5, we study stability of the scheme, and we will show that our scheme is stable for any step size $k$ and $h$ with the condition (1.3).

\section{Difference Scheme}

In the same way as in [2], we will approximate (1.1) by replacing the derivative for space and time in the difference operator

$$
\begin{gathered}
\frac{\partial}{\partial x}\left(a(x, t) \frac{\partial u(x, t)}{\partial x}\right) \cong \frac{1}{h^{2}} \delta(a(x, t) \delta u(x, t)), \\
\frac{\partial u(x, t)}{\partial x} \cong \frac{1}{h} \triangle u(x, t), \frac{1}{h} \nabla u(x, t),
\end{gathered}
$$




$$
\frac{\partial u(x, t)}{\partial t} \cong \frac{1}{k} \triangle u(x, t), \frac{1}{k} \nabla u(x, t),
$$

where $\delta$ is the central difference operator, $\triangle$ forward difference operator, $\nabla$ backward difference operator. We divide $x$-space to $N_{1}$ points, $t$-space to $N_{2}$ points where $h$ and $k$ are the mesh size for $x$-space,t-space respectively. We denote the approximation to $(1.1)$ at the mesh point $(x, t)=(j h, n k)$

$$
u_{j}^{n} \cong u(j h, n k)
$$

We set the vector $\left\|U^{n}\right\|$ by

$$
\left\|U^{n}\right\|=\max \left\{\left|u_{j}^{n}\right| ; 0 \leq j \leq 1 / h\right\} .
$$

We define the difference approximation to (1.1) by the following scheme.

If $\left\|U^{n}\right\|<1$. Then we set

$$
u_{j}^{n+1}=u_{j}^{n}+\frac{k}{1+\hat{k} L_{1}} \Phi\left(u_{j-1}^{n}, u_{j}^{n}, u_{j+1}^{n}\right) .
$$

If $\left\|U^{n}\right\| \geq 1$. Then we set

$$
u_{j}^{n+1}=u_{j}^{n}+\frac{k}{1+\hat{k} L_{1}\left\|U^{n}\right\|} \Phi\left(u_{j-1}^{n}, u_{j}^{n}, u_{j+1}^{n}\right),
$$

where

$$
\Phi\left(u_{j-1}^{n}, u_{j}^{n}, u_{j+1}^{n}\right)=\left\{\eta(j h, n k) u_{j-1}^{n}+\rho(j h, n k) u_{j}^{n}+\tau(j h, n k) u_{j+1}^{n}\right\}
$$

with

$$
\begin{gathered}
\rho(j h, n k)=-\left\{\frac{1}{h^{2}}\left\{a_{j+\frac{1}{2}}(n k)+a_{j-\frac{1}{2}}(n k)\right\}-\frac{\left|b_{j}(n k)\right|}{h}+c_{j}(n k),\right. \\
\eta(j h, n k)=\frac{1}{h^{2}} a_{j-\frac{1}{2}}(n k)+\frac{1}{2 h}\left\{\left|b_{j}(n k)\right|-b_{j}(n k)\right\}, \\
\tau(j h, n k)=\frac{1}{h^{2}} a_{j+\frac{1}{2}}(n k)+\frac{1}{2 h}\left\{\left|b_{j}(n k)\right|+b_{j}(n k)\right\}, \\
\left(n=0,1, . ., N_{1}+1, j=0,1, . ., N_{2}+1\right)\left(h=\frac{1}{N_{1}+1}, k=\frac{t_{F}}{N_{2}+1}\right),
\end{gathered}
$$

and

$$
a_{j}(t)=a(j h, t), b_{j}(t)=b(j h, t), c_{j}(t)=c(j h, t),
$$




$$
\begin{aligned}
L_{1}= & \max _{0 \leq i \leq \frac{1}{h}, 0 \leq j \leq \frac{t_{F}}{k}}\left\{\left(\frac{1}{h^{2}}\right)\left\{a_{i+\frac{1}{2}}(j k)+a_{i-\frac{1}{2}}(j k)\right\}\right. \\
& \left.+\frac{1}{h}|b(i h, j k)|-c(i h, j k)\right\} .
\end{aligned}
$$

The step size $\hat{k}$ in $(2.2),(2.3)$ is defined by

$$
\hat{k}=k^{1+\rho} \cdot \quad(\rho>0)
$$

\section{Truncation Error}

We define the truncation error $T(j h, n k)$ of $(2.2),(2.3)$

$$
\begin{aligned}
T(j h, n k) & =u(j h,(n+1) k)-u(j h, n k) \\
- & \frac{k}{\left(1+\hat{k} L_{1}\left\|\tilde{U}^{n}\right\|\right)} \Phi(u((j-1) h, n k), u(j h, n k), u((j+1) h, n k)),
\end{aligned}
$$

where

$$
\left\|\tilde{U}^{n}\right\|=\max \left\{1,\left\|U^{n}\right\|\right\}
$$

By Taylor series expansions of the exact solution $u(j h, n k)$, we have

$$
\begin{array}{r}
T(j h, n k)=k u_{t}(j h, n k)+O\left(k^{2}\right) \\
-\frac{k}{\left(1+\hat{k} L_{1}\left\|\tilde{U}^{n}\right\|\right)}+\left\{a_{x}(j h, n k) u_{x}(j h, n k)+a(j h, n k) u_{x x}(j h, n k)\right. \\
\left.+b(j h, n k) \tilde{u}_{x}(j h, n k)+c(j h, n k)+\frac{h}{2}|b(j h, n k)| u_{x x}(j h, n k)+O\left(h^{2}\right)\right\} \\
=k u_{t}(j h, n k)-\frac{k}{\left(1+\hat{k} L_{1}\left\|\tilde{U}^{n}\right\|\right)}\left\{u_{x}(j h, n k)+\frac{h}{2}|b(j h, n k)| u_{x x}(j h, n k)\right. \\
\left.+O\left(h^{2}\right)\right\} . \quad(3.2
\end{array}
$$

From (3.2), we have

$$
\begin{aligned}
& T(j h, n k)=k\left\{\frac{\hat{k} L_{1} \| \tilde{U^{n} \|}}{\left(1+\hat{k} L_{1} \| \tilde{\left.U^{n} \|\right)} u_{t}(j h, n k)\right.}\right. \\
& \quad-\frac{1}{2} \frac{h}{\left(1+\hat{k} L_{1}\left\|\tilde{U^{n}}\right\|\right)}|b(j h, n k)| u_{x x}(j h, n k)+O\left(h^{2}\right\} .
\end{aligned}
$$


From (3.3), we have the result.

Theorem 1. The truncation error of the difference approximation (2.2), (2.3) to (1.1) is given by

$$
T(j h, n k)=k^{(1+\tilde{\rho})} w(j h, n k),
$$

where

$$
\begin{aligned}
& w(j h, n k)=\frac{k^{(\rho-\tilde{\rho})}\left(k L_{1}\right)\left\|\tilde{U}^{n}\right\|}{\left(1+\hat{k} L_{1}\left\|\tilde{U}^{n}\right\|\right)} u_{t}(j h, n k) \\
& \quad-\frac{1}{2 \sqrt{c}} \frac{k^{\left(\frac{1}{2}-\tilde{\rho}\right)}}{\left(1+\hat{k} L_{1}\left\|\tilde{U}^{n}\right\|\right)}|b(j h, n k)| u_{x x}(j h, n k)+O\left(h^{2}\right),
\end{aligned}
$$

with

$$
\tilde{\rho}=\min \left\{\rho, \frac{1}{2}\right\}
$$

\section{Convergence}

In this section, we study the convergence of the scheme (2.2), (2.3).

We set the approximation error by

$$
e(j h, n k)=u(j h, n k)-u_{j}^{n}
$$

We use the abbreviation's

$$
\begin{gathered}
e_{j}^{n}=e(j h, n h), \\
T(j, n)=T(j h, n h), \\
u(j, n)=u(j h, n k) .
\end{gathered}
$$

From (2.2), (2.3), (3.1), and (4.1), we have

$$
e_{j}^{n+1}=e_{j}^{n}+p \Phi\left(e_{j-1}^{n}, e_{j}^{n}, e_{j+1}^{n}\right)+T(j, n),
$$

with

$$
p=\frac{k}{1+\hat{k} L_{1}\left\|\tilde{U}^{n}\right\|} .
$$


We set the initial conditions of (4.3)

$$
\begin{gathered}
e_{j}^{0}=0, \\
e_{j}^{1}=T(j, 1) \quad(0<j<1 / h) .
\end{gathered}
$$

We use Taylor series expansions of $\Phi\left(e_{j-1}^{n}, e_{j}^{n}, e_{j+1}^{n}\right)$

$$
\begin{aligned}
\Phi\left(e_{j-1}^{n}, e_{j}^{n}, e_{j+1}^{n}\right)=a_{x}(j, n) e_{x}( & j, n)+a(j, n) e_{x x}(j, n) \\
& +b(j, n) e_{x}(j, n)+c(j, n) e(j, n)+O(h) .
\end{aligned}
$$

Let us denote

$$
\begin{aligned}
q[m, u(x, m)]= & a(x, m k) \frac{\partial^{2}}{\partial x^{2}} u(x, m)+a_{x}(x, m k) \frac{\partial}{\partial x} u(x, m) \\
& +b(x, m k) \frac{\partial}{\partial x} u(x, m)+c(x, m k) u(x, m) \quad(m=1,2,3, . .),
\end{aligned}
$$

where

$$
\begin{gathered}
u(x, 1)=T(x, k), \\
u(x, m)=\sum_{l=1}^{m} T(x, l k) \quad(m=2, \ldots, n)
\end{gathered}
$$

Then we have

$$
\begin{gathered}
q[1, u(x, 1)]=a(x, k) \frac{\partial^{2}}{\partial x^{2}} T(x, k)+a_{x}(x, k) \frac{\partial}{\partial x} T(x, k)+b(x, k) \frac{\partial}{\partial x} T(x, k) \\
+c(x, k) T(x, k), \\
q[2, u(x, 2)]=a(x, 2 k)\left(\frac{\partial^{2}}{\partial x^{2}} \sum_{l=1}^{2} T(x, l k)\right) \\
+\frac{\partial}{\partial x} a(x, 2 k)\left(\frac{\partial}{\partial x} \sum_{l=1}^{2} T(x, l k)\right)+b(x, 2 k)\left(\frac{\partial}{\partial x} \sum_{l=1}^{2} T(x, l k)\right)+c(x, 2 k)\left(\sum_{l=1}^{2} T(x, l k)\right), \\
+\frac{\partial}{\partial x} a(x, m k)\left(\frac{\partial}{\partial x} \sum_{l=1}^{m} T(x, l k)\right)+b(x, m k)\left(\frac{\partial}{\partial x} \sum_{l=1}^{m} T(x, l k)\right)+c(x, m)\left(\sum_{l=1}^{m} T(x, l k)\right),
\end{gathered}
$$




$$
\begin{aligned}
& q[2, q[1, u(x, 1)]]=a(x, 2 k)\left(\frac{\partial^{2}}{\partial x^{2}} q[1, u(x, 1)]\right)+\frac{\partial}{\partial x} a(x, 2 k)\left(\frac{\partial}{\partial x} q[1, u(x, 1)]\right) \\
& +b(x, 2 k)\left(\frac{\partial}{\partial x} q[1, u(x, 1)]\right)+c(x, 2 k) q[1, u(x, 1)], \\
& q[m, q[m-1, u(x, m-1)]]]=a(x, m k)\left(\frac{\partial^{2}}{\partial x^{2}} q[m-1, u(x, m-1)]\right) \\
& +\left(\frac{\partial}{\partial x} a(x, m k)\right)\left(\frac{\partial}{\partial x} q[m-1, u(x, m-1)]\right) \\
& +b(x, m k)\left(\frac{\partial}{\partial x} q[m-1, u(x, m-1)]\right)+c(x, m k) q[m-1, u(x, m-1)] .
\end{aligned}
$$

From (4.3), (4.6), (4.8), we have

$$
\begin{gathered}
e_{j}^{n}=\Sigma_{l=1}^{n} T(j, l)+\left\{p q[1, u(x, 1)]+p^{2} q[2, q[1, u(x, 1)]]+p^{3} q[3, q[2, q[1, u(x, 1)]]]\right. \\
\left.+p^{n-1} q[n-1, q[n-2, q[n-3,[\ldots \ldots[q[1, u(x, 1)] \ldots]]]]]\right\} \\
+\left\{p q[2, u(x, 2)]+p^{2} q[3, q[2, u(x, 2)]]+p^{3} q[4, q[3,[q[2, u(x, 2)]]]\right. \\
\left.+\ldots+p^{n-2} q[n-1, q[n-2,[\ldots[. q[2, u(x, 2)]] . .]] \ldots]\right\}+\ldots+p q[n-1, u(x, n-1)] .
\end{gathered}
$$

We assume

$$
\begin{gathered}
\left\|\frac{\partial^{l_{1}}}{\partial x^{l_{1}}} f_{1}(x, t)\right\| \leq K_{1} \quad\left(l_{1}=0,1,2\right) \\
\left\|\frac{\partial^{l_{2}}}{\partial x^{l_{2}}}\left(f_{2}(x, t)\left(\frac{\partial^{l_{1}}}{\partial x^{l_{1}}} f_{1}(x, t)\right)\right)\right\| \leq K_{1}^{2} \quad\left(l_{1}, l_{2}=0,1,2\right), \\
\left\|\frac{\partial^{l_{m}}}{\partial x^{l_{m}}}\left(f_{m}(x, t)\left(\frac{\partial^{l_{m-1}}}{\partial x^{l_{m-1}}} f_{m-1}(x, t)\left(\frac{\partial^{l_{m-2}}}{\partial x^{l_{m-2}}} f_{m-2}(x, t) \ldots\left(\frac{\partial^{l_{1}}}{\partial x^{l_{1}}} f_{1}(x, t)\right)\right)\right) \ldots\right)\right\| \\
((x, t) \in \Omega=\{(x, t) ; 0<x<1,0<t F\})\left(K_{1}: \text { constant }\right)\left(l_{1}, l_{2},, . l_{m}=0,1,2\right),
\end{gathered}
$$

where

$f_{i}(x, t)(i=1,2, . ., m)$ are one of the functions $\{a(x, t), b(x, t), c(x, t)\}$.

We assume

$$
\left|\frac{\partial^{l}}{\partial x^{l}} w(x, t)\right| \leq \tilde{w}(x, t),
$$




$$
\begin{gathered}
\|\tilde{w}(x, t)\| \leq K_{1} . \\
(l=0,1,2, \ldots) \quad(x, t) \in \Omega=\left\{(x, t) ; 0<x<1,0<t \leq t_{F}\right\} .
\end{gathered}
$$

We use the following notation

$$
\tilde{w}(j, l)=\tilde{w}(j h, l k) .
$$

Then (4.9), (4.10), and (4.11), we have

$$
\begin{array}{r}
\left|e_{j}^{n}\right| \leq \Sigma_{l=1}^{n} k^{(1+\tilde{\rho})} \tilde{w}(j, l)+4 K_{1} p \Sigma_{l=0}^{(n-2)} r^{l} \tilde{w}(j, l)+4 K_{1} p \Sigma_{l=0}^{(n-3)} r^{l} \tilde{w}(j, l) \\
+\ldots+4 K_{1} p \Sigma_{l=1}^{(n-1)} \tilde{w}(j, l) \\
=\Sigma_{l=1}^{n} k^{(1+\tilde{\rho})} \tilde{w}(j, l)+4 K_{1} p\left\{\left(1-r^{n-1}\right)+\left(1-r^{n-2}\right) \Sigma_{l=1}^{2} \tilde{w}(j, l)\right. \\
+\ldots+(1-r) \Sigma_{l=1}^{(n-1)} \tilde{w}(j, l),
\end{array}
$$

with

$$
r=9 p K_{1}
$$

We set the maximum norm of the function $\tilde{w}(j, l)$

$$
\left\|W^{n}\right\|=\max _{1 \leq j \leq 1 / h}\{\tilde{w}(j, n)\}
$$

We have, after some computation,

$$
\begin{aligned}
\left|e_{j}^{n}\right| \leq \sum_{l=1}^{n} k^{(1+\tilde{\rho})}\left\|W^{n}\right\| & \\
& +\left\{2 K_{1} p n(n-1)-\frac{4 K_{1} p}{(r-1)^{2}}\left\{\frac{r^{(n+1)}-r^{2}}{(r-1)}-(n-1) r\right\}\right\}\left\|W^{n}\right\| .
\end{aligned}
$$

Then,we have

$$
\left|e_{j}^{n}\right| \leq n k^{(1+\tilde{\rho})}\left\|W^{n}\right\|+k^{(1+\tilde{\rho})}\left\{\frac{2 K_{1} p n^{2}}{(1-r)}+\frac{4 K_{1} p}{(1-r)^{3}} r^{2}+\frac{4 K_{1} p}{(1-r)^{2}} n r\right\}\left\|W^{n}\right\| .
$$

From (1.2), (4.4), we have

$$
\begin{gathered}
k n \leq t_{F}, \\
p n \leq \frac{t_{F}}{\left(1+k L_{1}\left\|\tilde{U}^{n}\right\|\right)}, \\
k^{1+\tilde{\rho}} p n^{2}\left\|W^{n}\right\| \leq\left(t_{F}\right)^{2} k^{\tilde{\rho}}\left\{\frac{\left\|W^{n}\right\|}{\left(1+\hat{k} L_{1}\left\|\tilde{U^{n}}\right\|\right)}\right\} .
\end{gathered}
$$


From (4.11), (4.15), and (4.16), we obtain the inequality

$$
\begin{aligned}
\left|e_{j}^{n}\right| \leq\left\{t_{F}+\frac{1}{(1-r)}\left(\frac{2 r t_{F}^{2}}{\left(1+\hat{k} L_{1}\left\|U^{n}\right\|\right)}\right.\right. & \\
& \left.\left.\quad \frac{4 p r^{3} k}{(1-r)^{2}}+\frac{4 r^{2} t_{F} k}{(1-r)\left(1+\hat{k} L_{1}\left\|U^{n}\right\|\right)}\right)\right\} k^{\tilde{\rho}} K_{1} .
\end{aligned}
$$

We set the maximum error of $\left\{e_{j}^{n}\right\}$ by

$$
\left\|E^{n}\right\|=\max _{1 \leq j \leq 1 / h}\left\{\left|e_{j}^{n}\right|\right\} .
$$

Then, from (4.17) we have

$$
\begin{aligned}
\left\|E^{n}\right\| \leq\left\{t_{F}+\frac{1}{(1-r)}(\right. & \frac{2 r t_{F}^{2}}{\left(1+\hat{k} L_{1}\left\|U^{n}\right\|\right)} \\
& \left.\left.+\frac{4 p r^{3} k}{(1-r)^{2}}+\frac{4 r^{2} t_{F} k}{(1-r)\left(1+\hat{k} L_{1}\left\|U^{n}\right\|\right)}\right)\right\} k^{\tilde{\rho}} K_{1} .
\end{aligned}
$$

From (4.18), we have

$$
\lim _{k->0}\left\|E^{n}\right\|=0 .
$$

Theorem [2] We consider the convergence paths

$$
\left\{\left(h_{i}, k_{i}\right) ; \frac{k_{i}}{h_{i}{ }^{2}}=c(c: \text { constant }) i=1,2, . ., h_{i}, k_{i} \rightarrow 0(i \rightarrow \infty)\right\} .
$$

Suppose that for $h_{i}$ and $k_{i}$, there exists positive numbers $j\left(h_{i}\right)$ and $n\left(k_{i}\right)$ such that

$$
j\left(h_{i}\right) h_{i} \rightarrow x \in[0,1](i \rightarrow \infty) \quad n\left(k_{i}\right) k_{i} \rightarrow t \in\left[0, t_{F}\right] .
$$

If the conditions (4.10), (4.11) and $|u(x, t)|,\left|u_{x}(x, t)\right|,\left|u_{x x}(x, t)\right|$ are bounded in $[0,1] *\left[0, t_{F}\right]$.

Then the scheme $(2.2),(2.3)$ converge to the solution $u(x, t)$ of the differential equation (1.1) uniformly.

\section{Stability}

In this section, we study the stability of the numerical process (2.2), (2.3) and define as follows. 
Definition. The numerical processes $\left\{Y^{n} \in R_{n}\right\}$ is stable if there exists a positive constant $K_{2}$ such that

$$
\left\|Y^{n}\right\| \leq K_{2}
$$

where $\|$.$\| denotes some norm and the constant K_{2}$ is depends only on initial value. mann.

We prove that the scheme (2.2), (2.3) are stable in mean of the von Neu-

We use the following Lemma in proof of stability.

\section{Lemma 1.}

$$
\left\|\Phi\left(e_{j-1}^{n}, e_{j}^{n}, e_{j+1}^{n}\right)\right\| \leq L_{2}, \quad\left(L_{2}: \text { constant }\right)
$$

under the conditions (4.10), (4.11).

Proof. From (2.4), we have

$$
\begin{gathered}
\Phi\left(e_{j-1}^{n}, e_{j}^{n}, e_{j+1}^{n}\right)=\frac{1}{h^{2}} a(j h, n k)\left(e_{j-1}^{n}-2 e_{j}^{n}+e_{j+1}^{n}\right)+\frac{1}{2 h} a_{x}(j h, n k)\left(e_{j+1}^{n}-e_{j-1}^{n}\right) \\
+\frac{1}{8} a_{x x}(j h, n k)\left(e_{j-1}^{n}-2 e_{j}^{n}+e_{j+1}^{n}\right)+\frac{1}{2 h}\left\{|b(j h, n k)|\left(e_{j-1}^{n}-2 e_{j}^{n}+e_{j+1}^{n}\right)\right. \\
\left.+b(j h, n k)\left(e_{j+1}^{n}-e_{j-1}^{n}\right)\right\}+c(j h, n k) T(j h, n k) \\
=a(j h, n k) e_{x x}(j h, n k)+a_{x}(j h, n k) e_{x}(j h, n k)+\frac{h^{2}}{8} a_{x x}(j h, n k) e_{x x}(j h, n k) \\
+\frac{h}{2}|b(j h, n k)| e_{x x}(j h, n k)+b(j h, n k) e_{x}(j h, n k)+c(j h, n k) e(j h, n k) .
\end{gathered}
$$

If we set the term of expansion $e_{j}^{n}$ by $\left\{s_{m_{1}}^{m_{2}}\right\}$. Then from (4.9), we have

$$
s_{m_{1}}^{m_{2}}=q\left[m_{2}-1, q\left[m_{2}-2,\left[\ldots, q\left[m_{1}, u\left(x, m_{1}\right)\right]\right]\right] . .\right] . \quad\left(m_{1}, m_{2}=1,2, . ., n\right)
$$

The factor of $s_{m_{1}}^{m_{2}}$ is given by

$$
\begin{aligned}
s(x, t)=f_{m_{2}}(x, t) \frac{\partial^{l_{s_{1}}}}{\partial x^{l_{1}}} & \left(f _ { m _ { 2 } - 1 } ( x , t ) \left(\frac{\partial^{l_{s_{2}}}}{\partial x^{l_{2}}} f_{m_{2}-2}(x, t)\right.\right. \\
& \left.\left.\left.\ldots\left(\frac{\partial^{l_{s_{p}}}}{\partial x^{l_{s_{p}}}} f_{m_{1}}(x, t)\right)\right)\right) \ldots\right) \frac{\partial^{l_{s_{q}}}}{\partial x^{l}}\left(\Sigma_{s_{q}}^{m_{1}} \tilde{T}(x, t)\right) .
\end{aligned}
$$

The first derivation of $s(x, t)$ is 


$$
\begin{gathered}
\frac{\partial}{\partial x} s(x, t)=\frac{\partial}{\partial x}\left(f _ { m _ { 2 } } ( x , t ) \frac { \partial ^ { l _ { s _ { 1 } } } } { \partial x ^ { l _ { s _ { 1 } } } } \left(f _ { m _ { 2 } - 1 } ( x , t ) \left(\frac{\partial^{l_{s_{2}}}}{\partial x^{l_{s_{2}}}} f_{m_{2}-2}(x, t)\right.\right.\right. \\
\left.\left.\left.\quad \ldots\left(\frac{\partial^{l_{s}}}{\partial x^{l_{s p}}} f_{m_{1}}(x, t)\right)\right)\right) \ldots\right) \frac{\partial^{l_{s_{q}}}}{\partial x^{l_{s_{q}}}}\left(\Sigma_{l=1}^{m_{1}} T(j, l)\right) \\
+f_{m_{2}}(x, t) \frac{\partial^{l_{s_{1}}}}{\partial x^{l_{s_{1}}}}\left(f _ { m _ { 2 } - 1 } ( x , t ) \left(\frac{\partial^{l_{s_{2}}}}{\partial x^{l_{s_{2}}}} f_{m_{2}-2}(x, t)\right.\right. \\
\left.\left.\left.\ldots\left(\frac{\partial^{l_{s_{p}}}}{\partial x^{l_{s_{p}}}} f_{m_{1}}(x, t)\right)\right)\right) \ldots\right) \frac{\partial^{l_{s_{q}}+1}}{\partial x^{l_{s_{q}}+1}}\left(\Sigma_{l=1}^{m_{2}} T(j, l)\right) .
\end{gathered}
$$

The second deraivation of $s(x, t)$ is

$$
\begin{aligned}
& \frac{\partial^{2}}{\partial x^{2}} s(x, t)= \frac{\partial^{2}}{\partial x^{2}}\left(f _ { m _ { 2 } } ( x , t ) \frac { \partial ^ { l _ { 1 } } } { \partial x ^ { l _ { s _ { 1 } } } } \left(f _ { m _ { 2 } - 1 } ( x , t ) \left(\frac{\partial^{l_{s_{2}}}}{\partial x^{l_{s_{2}}}} f_{m_{2}-2}(x, t)\right.\right.\right. \\
&\left.\left.\left.\ldots\left(\frac{\partial^{l_{s_{p}}}}{\partial x^{l_{s p}}} f_{m_{1}}(x, t)\right)\right)\right) \ldots\right) \frac{\partial^{l_{s_{q}}}}{\partial x^{l_{s_{q}}}}\left(\Sigma_{l=1}^{m_{1}} T(j, l)\right) \\
&+ 2 \frac{\partial}{\partial x}\left(f _ { m _ { 2 } } ( x , t ) \frac { \partial ^ { l _ { s _ { 1 } } } } { \partial x ^ { l _ { s _ { 1 } } } } \left(f _ { m _ { 2 } - 1 } ( x , t ) \left(\frac{\partial^{l_{s_{2}}}}{\partial x^{l_{s_{2}}}} f_{m_{2}-2}(x, t)\right.\right.\right. \\
&\left.\left.\left.\ldots\left(\frac{\partial^{l_{s p}}}{\partial x^{l_{s p}}} f_{m_{1}}(x, t)\right)\right)\right) \ldots\right) \frac{\partial^{l_{s_{q}}+1}}{\partial x^{l_{s_{q}}+1}}\left(\Sigma_{l=1}^{m_{1}} T(j, l)\right) \\
&+\left(f _ { m _ { 2 } } ( x , t ) \frac { \partial ^ { l _ { s _ { 1 } } } } { \partial x ^ { l _ { s _ { 1 } } } } \left(f _ { m _ { 2 } - 1 } ( x , t ) \left(\frac{\partial^{l_{s_{2}}}}{\partial x^{l_{s_{2}}}} f_{m_{2}-2}(x, t)\right.\right.\right. \\
&\left.\left.\left.\ldots\left(\frac{\partial^{l_{s_{p}}}}{\partial x^{l_{s_{p}}}} f_{m_{1}}(x, t)\right)\right)\right) \ldots\right) \frac{\partial^{l_{s_{q}}+2}}{\partial x^{l_{s_{q}}+2}}\left(\Sigma_{l=1}^{m_{1}} T(j, l)\right) .
\end{aligned}
$$

From (5.3), (5.4), we have

$$
\begin{gathered}
\left\|\frac{\partial}{\partial x} s_{m_{1}}^{m_{2}}\right\| \leq 2 \times 4 \times(7)^{\left(m_{2}-m_{1}\right)} K^{\left(m_{2}-m_{1}+1\right)}\left(\Sigma_{l=1}^{m_{1}}\left\|\frac{\partial^{l}}{\partial x^{l}} \tilde{T}(x, l)\right\|\right), \\
\left\|\frac{\partial^{2}}{\partial x^{2}} s_{m_{1}}^{m_{2}}\right\| \leq 4 \times 4 \times(7)^{\left(m_{2}-m_{1}\right)} K^{\left(m_{2}-m_{1}+1\right)}\left(\Sigma_{l=1}^{m_{1}}\left\|\frac{\partial^{l}}{\partial x^{l}} \tilde{T}(x, l)\right\|\right)
\end{gathered}
$$

Then, in the same way as (4.17), we have

$$
\begin{aligned}
& \left\|\frac{\partial}{\partial x} e(j, n)\right\| \leq 2 L_{n}, \\
& \left\|\frac{\partial^{2}}{\partial x^{2}} e(j, n)\right\| \leq 4 L_{n}
\end{aligned}
$$


with

$$
L_{n}=\left\{t_{F}+\frac{1}{(1-r)}\left(\frac{2 r t_{F}^{2}}{\left(1+\hat{k} L_{1}\left\|U^{n}\right\|\right)}+\frac{4 p r^{3} k}{(1-r)^{2}}+\frac{4 r^{2} t_{F} k}{(1-r)\left(1+\hat{k} L_{1}\left\|U^{n}\right\|\right)}\right)\right\} k^{\tilde{\rho}}\left\|W^{n}\right\| .
$$

From (5.2), (5.5), (5.6), we have

$$
\begin{array}{r}
\left\|\Phi\left(e_{j-1}^{n}, e_{j}^{n}, e_{j+1}^{n}\right)\right\| \leq\left\|a(j h, n k) e_{x x}(j h, n k)\right\|+\left\|a_{x}(j h, n k) e_{x}(j h, n k)\right\| \\
+\frac{h}{2}\left\|b(j h, n k) e_{x x}(j h, n k)\right\|+\left\|b(j h, n k) e_{x}(j h, n k)\right\|+\|c(j h, n k) e(j h, n k)\|+O\left(h^{2}\right) \\
\leq L_{2} . \quad\left(L_{2}=K_{1}\left(9+\frac{h}{2}\right) L_{n}\right)
\end{array}
$$

If we assume

$$
\begin{gathered}
\|u(x, t)\| \leq K_{3},\left\|u_{x}(x, t)\right\| \leq K_{3},\left\|u_{x x}(x, t)\right\| \leq K_{3}, \quad\left(K_{3}: \text { constant }\right), \\
(x, t) \in \Omega=\left\{(x, t) ; 0<x<1,0<t \leq t_{F}\right\} .
\end{gathered}
$$

Then, in the same way to (5.7), we have

$$
\begin{aligned}
&\|\Phi(u(j-1, n), u(j, n), u(j+1, n))\| \\
& \quad \leq\left\|a_{x}(j, n) u_{x}(j, n)\right\|+\left\|a(j, n) u_{x x}(j, n)\right\| \\
&+\left\|b(j, n) u_{x}(j, n)\right\|+\|c(j, n) u(j, n)\|+\frac{h}{2}\left(\| b(j, n) u_{x x}(j, n)\right) \| \\
& \quad \leq\left(4+\frac{h}{2}\right) K_{1} K_{3} .
\end{aligned}
$$

From (5.7), (5.9), we obtain:

$$
\begin{aligned}
&\left\|\Phi\left(u_{j-1}^{n}, u_{j}^{n}, u_{j+1}^{n}\right)\right\| \leq\|\Phi(u(j-1, n), u(j, n), u(j+1, n))\|+\left\|\Phi\left(e_{j-1}^{n}, e_{j}^{n}, e_{j+1}^{n}\right)\right\| \\
& \leq L_{3} . \quad\left(L_{3}=\left(4+\frac{h}{2}\right) K_{1} K_{3}+K_{1}\left(9+\frac{h}{2}\right) L_{n}\right)
\end{aligned}
$$

From (5.10), we have the following result.

\section{Lemma 2.}

$$
\left\|\Phi\left(u_{j-1}^{n}, u_{j}^{n}, u_{j+1}^{n}\right)\right\| \leq L_{3}, \quad\left(L_{3}: \text { constant }\right)
$$

with

$$
L_{3}=\left(4+\frac{h}{2}\right) K_{1} K_{3}+K_{1}\left(9+\frac{h}{2}\right) L_{n},
$$


with the condition (4.10), (4.11), (5.8).

From (2.2), (2.3), we have

$$
\begin{aligned}
u_{j}^{n+1}= & u_{j}^{n}+\frac{k}{1+\hat{k} L_{1}\left\|\tilde{U}^{n}\right\|} \Phi\left(u_{j-1}^{n}, u_{j}^{n}, u_{j+1}^{n}\right) \\
= & u_{j}^{n}+\frac{k}{1+k L_{1}} \Phi\left(u_{j-1}^{n}, u_{j}^{n}, u_{j+1}^{n}\right)+\frac{k}{1+\hat{k} L_{1}\left\|\tilde{U}^{n}\right\|} \Phi\left(u_{j-1}^{n}, u_{j}^{n}, u_{j+1}^{n}\right) \\
& -\frac{k}{1+k L_{1}} \Phi\left(u_{j-1}^{n}, u_{j}^{n}, u_{j+1}^{n}\right) .
\end{aligned}
$$

From (5.12), we have

$$
\begin{gathered}
\left|u_{j}^{n+1}\right| \leq\left|u_{j}^{n}+\frac{k}{1+k L_{1}} \Phi\left(u_{j-1}^{n}, u_{j}^{n}, u_{j+1}^{n}\right)\right|+\mid \frac{k}{1+\hat{k} L_{1}\left\|\tilde{U}^{n}\right\|} \Phi\left(u_{j-1}^{n}, u_{j}^{n}, u_{j+1}^{n}\right) \\
\quad-\frac{k}{1+k L_{1}} \Phi\left(u_{j-1}^{n}, u_{j}^{n}, u_{j+1}^{n}\right) \mid .
\end{gathered}
$$

We have the inequality

$$
\begin{gathered}
\left|u_{j}^{n}+\frac{k}{1+k L_{1}} \Phi\left(u_{j-1}^{n}, u_{j}^{n}, u_{j+1}^{n}\right)\right| \leq\left|1+\frac{k \rho(n h, j k)}{1+k L_{1}}\right|\left|u_{j}^{n}\right|+\frac{k}{1+k L_{1}} \eta(j h, n k)\left|u_{j-1}^{n}\right| \\
+\frac{k}{1+k L_{1}} \tau(j h, n k)\left|u_{j+1}^{n}\right| .
\end{gathered}
$$

From (2.5), we have

$$
1+\frac{k \rho(j h, n k)}{1+k L_{1}} \geq 0
$$

it follows

$$
\begin{gathered}
\left|u_{j}^{n}+\frac{k}{1+k L_{1}} \Phi\left(u_{j-1}^{n}, u_{j}^{n}, u_{j+1}^{n}\right)\right| \\
\leq\left\{1+\frac{k}{1+k L_{1}}\{\rho(n h, j k)+\eta(j h, n k)+\tau(j h, n k)\}\right\}\left\|U^{n}\right\| \\
\leq\left\|U^{n}\right\| .
\end{gathered}
$$

We study stability of the scheme (2.2). From condition (2.2), we have

$$
\frac{k}{1+\hat{k} L_{1}\left\|U^{n}\right\|} \Phi\left(u_{j-1}^{n}, u_{j}^{n}, u_{j+1}^{n}\right)-\frac{k}{1+k L_{1}\left\|\tilde{U}^{n}\right\|} \Phi\left(u_{j-1}^{n}, u_{j}^{n}, u_{j+1}^{n}\right)
$$




$$
=k \Phi\left(u_{j-1}^{n}, u_{j}^{n}, u_{j+1}^{n}\right)\left\{\frac{1}{\left(1+k L_{1}\left\|\tilde{U^{n}}\right\|\right)}-\frac{1}{\left(1+k L_{1}\right)}\right\} .
$$

Then, from (5.16), we have the inequality,

$$
\begin{gathered}
\left\|\frac{k}{1+\hat{k} L_{1}\left\|U^{n}\right\|} \Phi\left(u_{j-1}^{n}, u_{j}^{n}, u_{j+1}^{n}\right)-\frac{k}{1+k L_{1}\left\|\tilde{U}^{n}\right\|} \Phi\left(u_{j-1}^{n}, u_{j}^{n}, u_{j+1}^{n}\right)\right\| \\
\leq k\left\|\Phi\left(u_{j-1}^{n}, u_{j}^{n}, u_{j+1}^{n}\right)\right\|\left|\frac{1}{\left(1+\hat{k} L_{1}\left\|\tilde{U}^{n}\right\|\right)}-\frac{1}{\left(1+k L_{1}\right)}\right|,
\end{gathered}
$$

and

$$
\left|\frac{1}{\left(1+\hat{k} L_{1}\right)}-\frac{1}{\left(1+k L_{1}\left\|\tilde{U}^{n}\right\|\right)}\right| \leq 2 .
$$

Then, from (5.13), (5.14), (5.16), (5.17), we have

$$
\left|u_{j}^{n+1}\right| \leq\left|u_{j}^{n}\right|+2 k L_{3},
$$

From (5.18), we have

$$
\left|u^{n}\right| \leq\left|u_{i}^{0}\right|+2 n k L_{3}
$$

From (5.19), we have

$$
\left\|U^{n}\right\| \leq\left\|U^{0}\right\|+2 n k L_{3} \leq\left\|U^{0}\right\|+2 t_{F} L_{3} .
$$

We study stability of the scheme (2.3). From condition (2.3), we have

$$
\frac{k}{\left(1+\hat{k} L_{1}\left\|\tilde{U}^{n}\right\|\right)}=\frac{k}{\left(1+\hat{k} L_{1}\left\|U^{n}\right\|\right)} .
$$

From (5.12), we have the inequality

$$
\begin{gathered}
\left|u_{j}^{n+1}\right| \leq\left|u_{j}^{n}+\frac{k}{1+k L_{1}\left\|U^{n}\right\|} \Phi\left(u_{j-1}^{n}, u_{j}^{n}, u_{j+1}^{n}\right)\right| \\
+\left|\frac{k}{1+\hat{k} L_{1}\left\|U^{n}\right\|} \Phi\left(u_{j-1}^{n}, u_{j}^{n}, u_{j+1}^{n}\right)-\frac{k}{1+k L_{1}\left\|U^{n}\right\|} \Phi\left(u_{j-1}^{n}, u_{j}^{n}, u_{j+1}^{n}\right)\right| .
\end{gathered}
$$

From the condition $\left\|U^{n}\right\| \geq 1$, we have

$$
1+\frac{k \rho(j h, n k)}{1+k L_{1}\left\|U^{n}\right\|} \geq 0 .
$$

In the same way to $(5.15)$, we have

$$
\left|u_{j}^{n}+\frac{k}{1+k L_{1}} \Phi\left(u_{j-1}^{n}, u_{j}^{n}, u_{j+1}^{n}\right)\right| \leq\left\|U^{n}\right\| .
$$


From Lemma [2], we have

$$
\begin{gathered}
\left|\frac{k}{1+\hat{k} L_{1}\left\|U^{n}\right\|} \Phi\left(u_{j-1}^{n}, u_{j}^{n}, u_{j+1}^{n}\right)-\frac{k}{1+k L_{1}\left\|U^{n}\right\|} \Phi\left(u_{j-1}^{n}, u_{j}^{n}, u_{j+1}^{n}\right)\right| \\
\leq \mid k \Phi\left(u_{j-1}^{n}, u_{j}^{n}, u_{j+1}^{n}\right) \frac{|(k-\hat{k})| L_{1}\left\|U^{n}\right\|}{\left(1+k L_{1}\left\|U^{n}\right\|\right)\left(1+\hat{k} L_{1} \| U^{n}\right) \| \mid} \\
\leq\left\{k L_{1} L_{3}+O(k \sqrt{k})\right\}\left\|U^{n}\right\| .
\end{gathered}
$$

From (5.21), (5.22), (5.23), we have

$$
\left|u_{n+1}^{j}\right| \leq\left(1+k L_{1} L_{3}+O(k \sqrt{k})\right)\left\|U^{n}\right\|,
$$

and we have the following result

$$
\left\|U^{n+1}\right\| \leq\left\{1+k L_{1} L_{3}+O(k \sqrt{k})\right\}^{n}\left\|U^{0}\right\| .
$$

From (5.24), we have

$$
\left\|U^{n}\right\| \leq e^{w_{1} t_{F}}\left\|U^{0}\right\|
$$

with

$$
w_{1}=L_{1} L_{3} .
$$

Summarizing the results, we have

Theorem [3] For any given step size $h, k$, we set the constant $\hat{k}, L_{1}$ to be

$$
\begin{gathered}
L_{1}=\max _{0 \leq i \leq \frac{1}{h}, 0 \leq j \leq \frac{t_{F}}{k}}\left\{\left(\frac{1}{h^{2}}\right)\left\{a_{i+\frac{1}{2}}(j k)+a_{i-\frac{1}{2}}(j k)\right\}+\frac{1}{h}|b(i h, j k)|-c(i h, j k)\right\} \\
\hat{k}=k^{1+\alpha}, \quad(\alpha>0)
\end{gathered}
$$

and if $|u(x, t),| u_{x}(x, t) \mid$, and $\left|u_{x x}(x, t)\right|$ are bounded in the region

$\left.(x, t) \in \Omega=\left\{(x, t) ; 0<x<1,0<t \leq t_{F}\right\}\right)$ and the conditions (4.10), (4.11) are satisfied. Then the difference approximation (2.2), (2.3) are stable.

\section{References}

[1] E.G. Du Fort, E.C. Frankel, Stability conditions in the numerical treatment on parabolic differential equations, Math. Tables and other Aids to Computation, 17 (1953), 135. 
[2] M. Nakashima, Unconditionally stable explicit difference schemes for the variable coefficients parabolic differential equation (II), In: Processing Techniques and Applications International Conference, Las Vegas, Nevada,USA June (2001); Proceedings of the International Conference on Parallel and Distributed Processing (2001),561-569.

[3] M. Nakashima, Unconditionally Stable Explicit Difference Schemes for the Variable Coefficients Parabolic Differential Equation (IV), Numerical Methods and Applications, Lecture Notes In Computer Sciece, Ed-s: Dinov et al, Springer, 2542 (2002), 536-544.

[4] M. Nakashima, Unconditionally stable explicit difference schemes for the variable coefficients two dimensional parabolic differential equation (V), Journal of Applied Mechanics, 54 (2003), 327-341.

[5] W.E. Schiesser, The Numerical Methods of Lines, Academic Press, San Diego (1991).

[6] R.D. Richtmyer, D.K. Morton, Difference Methods for Intial-Value Problems, Wiley, New York (1967). 